\title{
Burnout in Referees: Relations with Stress, Cognitive Appraisal, and Emotions \\ Rui Gomes $^{1,2^{*}}$, Liliana Fontes ${ }^{1}$, Marta Rodrigues ${ }^{2}$ and Belarmino Dias ${ }^{2}$ \\ ${ }^{1}$ Psychology Research Centre, School of Psychology, University of Minho, Portugal, Campus de Gualtar, 4710-057 Braga, Portugal \\ ${ }^{2}$ Adaptation, Performance, and Human Development Research Group, School of Psychology, University of Minho, Braga, Portugal, Campus de Gualtar, 4710-057 Braga, Portugal
}

\begin{abstract}
Background: Referees assume an important role on sports activity because their decisions are highly scrutinized and can influence the results achieved by athletes and teams. However, referees are much less studied than athletes regarding the way they deal with and respond to stress factors. This study analysed how soccer referees adapted to a stressful situation by using a critical incident methodology of collecting data 24 to 48 hours before a game. For that, we established two goals and five hypotheses: goal 1) to describe the feelings of stress, cognitive appraisal, emotions, and burnout in referees; goal 2) to analyse the predictor value of stress, cognitive appraisal, and emotions in explaining the referees' burnout experience. The five hypotheses tested were: higher levels of stress predict the referees' tendency to burnout (H1), higher levels of threat perception and lower levels of challenge perception predict the referees' tendency to burnout (H2); lower levels of coping perception and control perception predict the referees' tendency to burnout (H3), higher levels of anxiety, dejection, and anger predict the referees' tendency to burnout (H4), and lower levels of excitement and happiness predict the referees' tendency to burnout (H5).
\end{abstract}

\section{Publication History:}

Received: April 16, 2021

Accepted: May 06, 2021

Published: May 08, 2021

\section{Keywords:}

Burnout, Cognitive appraisal, Soccer referees, Sports emotions, Sports performance, Stress adaptation

Methods: The evaluation protocol included measures of stress, cognitive appraisal, emotions, and burnout. Participants were 394 soccer referees (males $=364 ; 90 \%)$, with a mean age of 27 years old $(S D=7.33)$.

Results: Results indicated that burnout was predicted by these dimensions: higher levels of stress related to conflicts and family and personal life balance, higher levels of threat perception, lower levels of challenge perception, lower levels of coping perception, lower levels of happiness, and higher levels of anger.

Conclusion: These results indicate that referees can benefit from participating in psychological programs that include stress management strategies directed to deal with the stress and negative emotions from competition.

\section{Adaptation to Stress in Soccer Referees: Relation with Burnout and Performance}

Referees assume an important role in sports because they can determine a game and an entire competition's result [1]. Therefore, actual errors (those actions referees take that are objectively proven wrong) or perceived errors (actions that other sports agents perceive as mistakes at the time, but that were in fact correct decisions) can be seen as unforgivable and may lead to verbal abuse, threats, or even physical aggression [2]. Research indicates that the referee activity can be stressful due the exposure to criticism and pressure from supporters, sports agents, and the media $[3,4]$. In this way, it becomes important to analyse how referees evaluate and cope with stressors resulting from their activity. This analysis should assume theoretical orientations from models explaining human adaptation to stress that reinforce the importance of considering not only the role of stressors and reactions to stress, but also the role played by cognitive appraisal and emotions during the adaptation process. For example, the well-known transactional stress model $[5,6]$ proposes that a potentially stressful event activates primary and secondary cognitive appraisals [5]. In the primary cognitive appraisal, the individual interprets the situation as either threatening or challenging, perceiving it as potentially generating loss or benefit [5]. In the secondary cognitive appraisal, the individual evaluates the coping processes available to deal with the events perceived as generating stress [6]. Therefore, a stress reaction is triggered when a personally significant activity within a sports context is perceived as too demanding, taking into account the personal resources referees believe they have to cope with stress situations. However, Gomes [7] reinforces that the adaptation to stress only begins if stressors are considered important and relevant to the individuals' wellbeing, which means that it can be very restrictive to analyse perceptions of threat or challenge if we do not first consider the cognitive appraisal of the event's importance to human functioning. Nevertheless, these cognitive appraisal processes activate emotional responses that can be understood as organized psychological reactions to continuous relationships with the environment [8]. Research points to the relation between challenge perception and pleasant emotions $[9,10]$, whereas a threat perception tends to result in unpleasant emotions [10]. Thus, adaptation to stress can be understood as an interactive process between a specific situation and the individual, involving a stressful event (or constellation of events), primary and secondary processes of cognitive appraisal, immediate reactions (such as emotions), and long term reactions or consequences of the stressful events (such as satisfaction, commitment, or even burnout).

Although it is evident that all these factors are involved in adaption in stress, there persists a lack of indications about how they relate to each other and how they influence the way individuals evaluate and cope with stress $[6,7,11,12]$. This lack of evidence is even more

"Corresponding Author: Prof. Rui Gomes, Universidade do Minho, Escola de Psicologia, Campus de Gualtar, 4710-057 Braga, Portugal, Phone: +351 253604 232, Fax: +351 253604 229; E-mail: rgomes@psi.uminho.pt

Citation: Gomes R, Fontes L, Rodrigues M, Dias B (2021) Burnout in Referees: Relations with Stress, Cognitive Appraisal, and Emotions. Int J Psychol Behav Anal 7: 177. doi: https://doi.org/10.15344/2455-3867/2021/177

Copyright: (C) 2021 Gomes et al. This is an open-access article distributed under the terms of the Creative Commons Attribution License, which permits unrestricted use, distribution, and reproduction in any medium, provided the original author and source are credited. 
evident in the referee activity because studies have typically analysed the adaptation process in a compartmented way. For example, the studies of Hill et al. [13] and Neil et al. [14] produced interesting and promising findings about stress, cognitive appraisal, coping, and emotional reactions in referees, but they were both qualitative, which limited the analysis of specific relations between variables. Ritchie and colleagues [1] adopted a critical incident methodology to study how referees perform when facing a wide range of stressors, but they restricted their analysis to primary cognitive appraisals.

In an attempt to integrate in a cohesive methodology the factors involved in referees' adaptation to stress, we analysed how stress factors, cognitive appraisal, and emotions could explain the referees' reactions to stress, measured in terms of burnout (i.e., feelings of emotional exhaustion, physical fatigue, and cognitive weariness, [15]). Specifically, a critical incident methodology was employed where the setting of human adaptation was a game that occurred in the following 24 to 48 hours, in order to analyse four variables involved in the adaption to stress: (1) the levels of stress generated by this sport event (game); (2) the processes of cognitive appraisal, including the importance given by the referees to the following game, the primary appraisals of threat and challenge, and the secondary appraisals of coping perception and coping perception; (3) the emotions felt by referees when they think about the next game; and (4) the levels of burnout that they felt in their lives at that moment. Thus, we proposed an integrated analysis starting from stress to cognitive appraisal and then to emotions in order to explain burnout feelings in referees. Since burnout occurs over time as a chronic response to stress [16], it is likely that one single data collection can capture it. Also, we measured burnout in our study by asking referees to consider the previous 30 days [17], meaning that we captured their burnout feelings in a relatively stable period of time. In sum, the methodology used in our study focuses on analysing an event that participants view as important to their wellbeing and then characterizing their cognitive and emotional responses before a game. For that, we used a critical incident methodology in order to consider the events' context and circumstances, which has as main advantages the flexibility of application to several situations, the focus on what is considered as more important and critical to participants, and its cost-effectiveness [18].

Considering the purpose of analysing the referees' adaptation to stress, we established as goals for this study the characterization of feelings of stress, cognitive appraisal, emotions, and burnout before a game (Goal 1) and, most important, the analysis of the predictor value of stress, cognitive appraisal, and emotions in explaining the referees' burnout experience (Goal 2). In order to clarify Goal 2, we established the following hypotheses:

H1. Higher levels of stress predict the referees' tendency to feel burnout.

H2. Higher levels of threat perception and lower levels of challenge perception predict the referees' tendency to feel burnout.

H3. Lower levels of coping perception and control perception predict the referees' tendency to feel burnout.

H4. Higher levels of anxiety, dejection, and anger predict the referees' tendency to feel burnout.

H5. Lower levels of excitement and happiness predict the referees' tendency to feel burnout.

\section{Material and Methods}

\section{Participants}

This study used a convenience non-probabilistic sampling method. Participants included 394 soccer referees working in Portugal, 29 (7.4\%) female and 364 (92.6\%) male, aged $17-48$ years old $(M=$ 26.53; $S D=7.33)$. Referees belonged to several categories from all competitive divisions: under $13(n=8,2.5 \%)$, under $15(n=34,8.6 \%)$, under $19(n=53,13.5 \%)$, over $19(n=69,17.5 \%)$, and adults $(n=228$, $57.9 \%)$, both at district $(n=325,82.5 \%)$ and national $(n=69,17.5 \%)$ levels. Participants had been refereeing for 1 to 30 years $(M=6.82$, $S D=5.55)$.

\section{Measures}

Demographic Questionnaire: Included questions on sex, age, category, competitive division, competitive rank, and years of refereeing practice in official competitions.

Referees Stress Questionnaire (RSQ; [19]): Evaluates the general level of stress in referees' activity with one item responded on a 5-point Likert-type scale ( $0=$ No stress at all; $4=$ Very high stress). Then, the instrument includes 20 items divided into five stress subscales: errors ( $\alpha$ for this study $=.89$ ), conflicts ( $\alpha$ for this study $=$ .80 ), poor sport condition ( $\alpha$ for this study $=.86$ ), sports career ( $\alpha$ for this study $=.84$ ), and family and personal life ( $\alpha$ for this study $=.81$ ). Items were evaluated on a five-point Likert-type scale with higher values indicating higher levels of stress in each dimension; an example item was "Commit serious mistakes during this game". This instrument has a general and a specific version. In this study the latter was used, where referees indicated their level of stress regarding the next competitive situation (in our case, the next game). The confirmatory factorial analysis (CFA) revealed good psychometric properties for this instrument $(\mathrm{c} 2(160 d f)=481.106, p<.001 ; \mathrm{CMIN} / \mathrm{DF}=3.007$; RMSEA $=.071,90 \%$ CI $[.064 ; .079]$; CFI $=.925 ; \mathrm{TLI}=.911)$ [20].

Primary and Secondary Cognitive Appraisal Scale (PSCAS; [21]): Evaluates primary and secondary appraisals according to the dimensions proposed by Lazarus [5,9]. Primary cognitive appraisal includes three subscales: importance perception ( $\alpha$ for this study $=.91$ ), threat perception ( $\alpha$ for this study $=.85$ ), and challenge perception ( $\alpha$ for this study $=.90$ ). Secondary cognitive appraisal includes coping perception ( $\alpha$ for this study $=.88$ ) and control perception ( $\alpha$ for this study $=.75$ ). The instrument includes 15 items (three for each subscale), such as "To what extent do you feel that you control what should be done in your work?", responded on a sevenpoint Likert-type scale, with higher values indicating higher scores on each subscale. For this study, we used the specific version of the instrument (i.e., related to the next game). The CFA revealed good psychometric properties for the instrument $(\mathrm{c} 2(80 d f)=206,332, \mathrm{p}<$ .001 ; $\mathrm{CMIN} / \mathrm{DF}=2.58$; RMSEA $=.063,90 \%$ CI $[.053 ; .074]$; CFI $=$ $.965 ; \mathrm{TLI}=.953)[20]$.

Sport Emotion Questionnaire (SEQ; Adapted from [22]): Evaluates the subjective feelings associated with five emotions related to sport competition: anxiety ( $\alpha$ for this study $=.88$ ), dejection ( $\alpha$ for this study $=.93$ ), anger ( $\alpha$ for this study $=.91)$, excitement ( $\alpha$ for this study $=.78$ ), and happiness ( $\alpha$ for this study $=.91$ ), totalling 22 items, which list emotions such as "excited". Items were measured on a five-point Likert-type scale, where higher values indicate greater emotional intensity (total values for each subscale between $0-4$ ). For this study 
we used the pre-competitive version of the instrument, which is answered by asking referees to think about how they feel about their next game. The CFA revealed good psychometric properties for the instrument $(\mathrm{c} 2(197 d f)=618.742, \mathrm{p}<.001$; CMIN / DF $=3.141$; RMSEA $=.074,90 \%$ CI $[.067 ; .080] ; \mathrm{CFI}=.938, \mathrm{TLI}=.928)[20]$.

Shirom-Melamed Burnout Measurement (SMBM; Adapted from [15]): This instrument evaluates burnout in three dimensions: physical fatigue ( $\alpha$ for this study $=.90$ ), cognitive weariness ( $\alpha$ for this study $=$ .94 ), and emotional exhaustion ( $\alpha$ for this study $=.88$ ). Referees were instructed to think about how they felt about their refereeing activity in the past month by using 14 items, such as "I feel tired", answered on a seven-point Likert-type scale, where higher values correspond to higher levels in each dimension (total values for each dimension range from 1 to 7 ). For this study scores $\geq 5$ were indicative of burnout [23]. The CFA revealed good psychometric properties for the instrument $(\mathrm{c} 2(72 d f)=193,122, \mathrm{p}<.001 ; \mathrm{CMIN} / \mathrm{DF}=2.682 ; \mathrm{RMSEA}=.065$, $90 \%$ CI $[.054 ; .077]$; CFI $=.974 ;$ TLI $=.967)[20]$.

\section{Procedure}

The study was conducted in accordance with the Declaration of Helsinki and was approved by the Ethics Commission of the authors' university (SECSH 016/2015). Referee's soccer associations were then contacted by our research team and four of them agreed to collaborate. Referees were then contacted to ask for their willingness to participate in the study. Those who agreed were informed about the study's objectives and procedure and signed the informed consent form. Questionnaires were answered 24 to 48 hours before the game and took approximately 15 minutes to complete. The referees answered the questionnaires in paper and delivered them directly to our team; they mostly filled the evaluation protocol at their training facilities; some of them took the questionnaires to complete at home and delivered them the following day.

\section{Analysis}

All data was analysed using SPSS (version 24). We used descriptive and correlation analyses to provide a better understanding of how variables were perceived by referees and how they relate to each other (Goal 1). Then, in order to analyse the processes of referees' adaptation to stress, we used linear regression analysis (with block procedures) to explain both the burnout experience and the perception of sport performance felt by referees 24 to 48 hours before a game (Goal 2 and Hypotheses 1 to 5). Regression analysis was selected because it allowed for predictor variables to be entered in the regression model as proposed by Gomes' [7] model: first, we introduced the stress variables, then the cognitive appraisal variables, followed by the emotional response variables (Figure 1). The importance perception of the PSCAS instrument was used to select participants that perceived the next game as minimally significant, since adaptation to stress implies that individuals perceive the situation as relevant to their well-being. Therefore, as in previous studies, a cut-off point was established in this PSCAS dimension where participants scoring two or lower on the Likert-type scale were removed from analyses [24], which was the case for 12 participants.

\section{Results}

\section{Descriptive statistics of variables}

Table 1 presents the global stress levels referees experienced before the competition, the most prevalent sources of stress, their cognitive appraisal, emotions, and burnout levels. Only approximately $10 \%$ of participants experienced no stress before the competition, with the majority reporting moderate levels of stress (43.5\%). On average, errors and sports career were the most prevalent sources of stress for referees. Regarding cognitive appraisal, coping perception and control perception emerged as the highest rated dimensions, whereas happiness and excitement were the emotions most reported

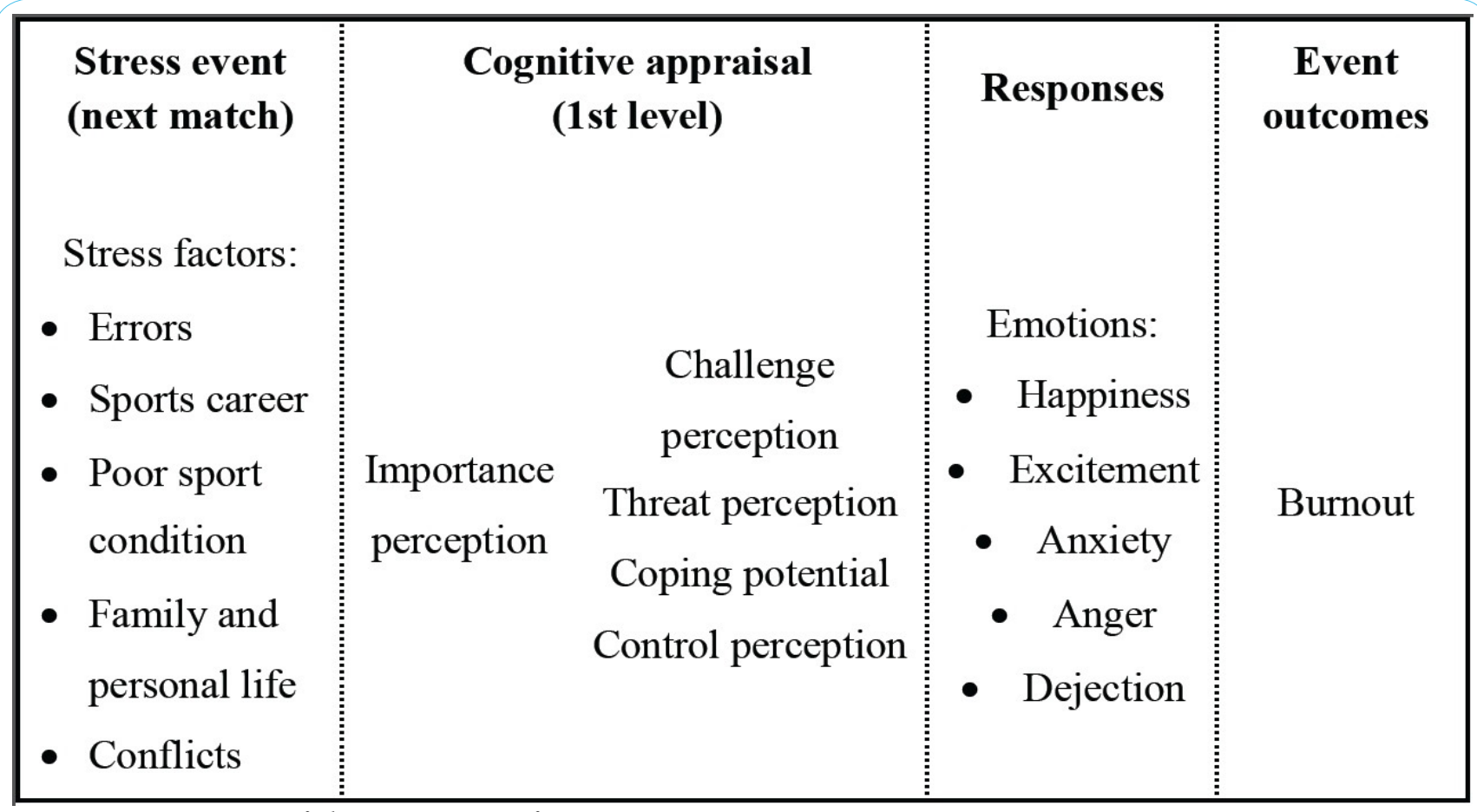

Figure 1: Interactive process of adaption to stress in referees. 
by referees. The dimensions that most contributed to burnout were physical fatigue and cognitive weariness. Regarding burnout, $2 \%$ of participants experienced burnout (global score), with $4.5 \%$ reporting physical fatigue problems (specific score).

\begin{tabular}{|c|c|}
\hline & Total sample \\
\hline RSQ: Global stress level (range 0-4) & $n(\%)$ \\
\hline No stress at all & $26(10.2)$ \\
\hline Not muchstress & $98(38.4)$ \\
\hline Some stress & $111(43.5)$ \\
\hline Much stress & $18(7.1)$ \\
\hline Very high stress & $2(0.8)$ \\
\hline RSQ: Stress factors (range 0-4) & $M(D P)$ \\
\hline Errors & $2.17(0.92)$ \\
\hline Sports career & $2.02(1.00)$ \\
\hline Poor sport condition & $1.55(0.99)$ \\
\hline Family and personal life & $1.47(0.94)$ \\
\hline Conflicts & $1.17(0.74)$ \\
\hline PSCAS: Cognitive appraisal (range 0-6) & $M(D P)$ \\
\hline Coping perception & $5.11(0.73)$ \\
\hline Control perception & $4.74(0.87)$ \\
\hline Importance perception & $4.62(1.21)$ \\
\hline Challenge perception & $4.53(1.22)$ \\
\hline Threat perception & $1.47(1.28)$ \\
\hline SEQ: Emotions (range 0-4) & $M(D P)$ \\
\hline Happiness & $1.37(1.06)$ \\
\hline Excitement & $1.05(0.91)$ \\
\hline Anxiety & $0.14(0.99)$ \\
\hline Anger & $0.03(1.12)$ \\
\hline Dejection & $0.02(1.17)$ \\
\hline SMBM: Burnout measure (range 1-7) & $M(D P)$ \\
\hline Physical fatigue & $2.56(1.16)$ \\
\hline Cognitive weariness & $2.02(1.04)$ \\
\hline Emotional exhaustion & $1.71(0.95)$ \\
\hline SMBM: Burnout levels (range 1-7) & $n(\%)$ \\
\hline Physical fatigue $\geq 5$ & $17(4.5)$ \\
\hline Cognitive weariness $\geq 5$ & $9(2.4)$ \\
\hline Emotional exhaustion $\geq 5$ & $7(1.8)$ \\
\hline Total Burnout $\geq 5$ & $8(2.1)$ \\
\hline
\end{tabular}

\section{Explaining burnout}

As referred, the variables selected for the regression were introduced in four blocks, according to Gomes' [7] interactive proposal (stress dimensions, primary cognitive appraisal, secondary cognitive appraisal, and emotions). Block 1 indicated that higher levels of stress related with family and personal life, as well as with higher levels of conflicts, predicted burnout, with the model explaining $14 \%$ of the variance. Block 2 indicated that higher levels of threat perception and lower levels of challenge perception predicted burnout, with the global model explaining $18 \%$ of the variance. Block 3 indicated that lower coping perception predicted burnout, with the global model explaining $20 \%$ of the variance. Finally, block 4 indicated that lower levels of happiness and higher levels of anger emerged as predictors of burnout, with the global model explaining $24 \%$ of the variance. Table 2 includes all the regression results.

\section{Discussion}

The main objective of this study was to analyse how referees experience and adapt to a stressful situation by explaining their feelings of burnout. This analysis was done by assuming an interactive approach that "joined together" the stressful event (sources of stress in referees $24 \mathrm{~h}$ to $48 \mathrm{~h}$ prior to a game), the processes of cognitive appraisal of the game, the emotional responses, and the final experience of burnout, adopting as background transactional and interactive models of human stress $[5-7,25,26]$. The conjunction of these variables permits a holistic understanding of how individuals adapt to stress [7], which allows a more comprehensive perspective of this topic than assuming separate approaches of analysis centred in one or two of these variables, most notably cognitive appraisal of coping or emotions in sports [27,28].

Starting by the characterization of feelings of stress, cognitive appraisal, emotions, and burnout before a game (Goal 1 of the study), our results indicated that, before a game, the majority of referees reported moderate levels of stress (43.5\%), which is corroborated by previous studies $[13,28,29]$. The main source of stress experienced by the referees was related to committing errors, that is, the possibility of making incorrect decisions in important moments of the game. Curiously, referees and athletes do not seem to be very different in this stress factor, because there are also indications that elite athletes suffer from fear of making mistakes [30]. The second source of stress referred by referees was sports career, with values close to committing errors. In this case, referees were most preoccupied with the evaluations made by observers recruited from referee committees about their performance during the game. This evaluation is very important in ranking the referees and can influence their progress or demotion to other sports divisions. In fact, we suppose that committing errors and sports career emerge as mains factors of stress because they are related on a daily basis for referees, i.e., if they have good performances in games, then the sports career factor can be mitigated; if they have poor performances, then it is expected that sports career will produce higher tension in referees. Another interesting aspect was the fact that, despite close to half our sample reporting moderate levels of stress, burnout was experienced by only $2.1 \%$ of referees, with the dimensions of physical fatigue and cognitive weariness contributing the most to this negative experience. Fortunately, the majority of referees do not seem to experience burnout, which is also in accordance with other studies [31-33]. Moreover, this result can be explained by the processes of cognitive appraisal that can interfere on the impact produced by stress on the referees' emotions and burnout.

This is confirmed by the second goal of our study that shows that stress factors are central predictors of burnout (explaining $14 \%$ of the burnout variance), but the processes of cognitive appraisal should not be disregarded because they increase the explained variance to $20 \%$, with emotions increasing the final regression model to $24 \%$. Thus, the combination of these three variables is important to explain the referees' experience. Analysing in more detail the factors explaining burnout, we verified that higher levels of stress related to balancing family and sports life and higher levels of conflicts are related to a greater propensity for burnout symptoms, partially corroborating our study's Hypothesis 1, because not all sources of 
Citation: Gomes R, Fontes L, Rodrigues M, Dias B (2021) Burnout in Referees: Relations with Stress, Cognitive Appraisal, and Emotions. Int J Psychol Behav Anal 7: 177. doi: https://doi.org/10.15344/2455-3867/2021/177

\begin{tabular}{|c|c|c|c|c|c|c|c|c|}
\hline & $\mathrm{R}^{2}\left(\mathrm{R}^{2} \mathrm{Aj}.\right)$ & $\Delta \mathrm{R}^{2}$ & $\Delta \mathrm{F}$ & $\mathrm{F}$ & $\beta$ & $\mathrm{t}$ & IT & VIF \\
\hline Block 1 & $.17(.14)$ & .15 & $7.45^{\star *}$ & $(8,209) 5.37^{\star \star}$ & & & & \\
\hline RSQ: Errors & & & & & -.18 & -1.86 & .41 & 2.45 \\
\hline RSQ: Sports career & & & & & .05 & 0.54 & .45 & 2.21 \\
\hline $\begin{array}{l}\text { RSQ: Poor sport } \\
\text { condition }\end{array}$ & & & & & .07 & 0.47 & .49 & 2.06 \\
\hline $\begin{array}{l}\text { RSQ: Family and } \\
\text { pers. life }\end{array}$ & & & & & .22 & $2.80^{\star *}$ & .64 & 1.57 \\
\hline RSQ: Conflicts & & & & & .25 & $3.20^{\star *}$ & .64 & 1.56 \\
\hline Block 2 & $.21(.18)$ & .04 & $5.59^{\star *}$ & $(10,207) 5.61^{\star *}$ & & & & \\
\hline PSCAS: Threat & & & & & .14 & $2.11^{*}$ & .89 & 1.13 \\
\hline PSCAS: Challenge & & & & & -.16 & $-2.43^{*}$ & .89 & 1.13 \\
\hline Block 3 & $.24(.20)$ & .03 & $3.69^{*}$ & $(12,205) 5.41^{\star *}$ & & & & \\
\hline PSCAS: Coping & & & & & -.17 & $-2.24^{*}$ & .65 & 1.54 \\
\hline PSCAS: Control & & & & & -.06 & -0.81 & .80 & 1.25 \\
\hline Block 4 & $.30(.24)$ & .06 & $3.42^{\star *}$ & $(17,200) 5.05^{\star *}$ & & & & \\
\hline SEQ: Anxiety & & & & & -.04 & -0.35 & .36 & 2.78 \\
\hline SEQ: Dejection & & & & & -.17 & -1.16 & .16 & 6.11 \\
\hline SEQ: Excitement & & & & & .04 & 0.40 & .37 & 2.73 \\
\hline SEQ: Anger & & & & & .34 & $2.16^{*}$ & .14 & 6.97 \\
\hline SEQ: Happiness & & & & & -.26 & $-2.75^{\star \star}$ & .38 & 2.63 \\
\hline
\end{tabular}

stress predicted burnout. The stress related to family and personal life can be explained by the fact that the majority of referees assume this activity in parallel with other professional activities that are their main sources of income. This can increase pressure towards balancing the referees' sports activity with their family roles. Moreover, refereeing typically occurs on weekends, which is a time mostly dedicated to family. Conflicts was another stress factor predictor of the burnout experience, meaning that referees should be prepared to deal as best they can with negative attitudes and behaviours from athletes, coaches, and even spectators during a game, because these negative and stressful situations seem to be "part of the game". Previous studies align with our results, pointing out time pressures, conflict, and fear of failure as predictors of burnout $[34,35]$. Cognitive appraisal also played an important role in the burnout experience because a higher tendency to experience burnout symptoms was related to increases in threat perception and decreases in challenge perception and coping perception, as predicted by theoretical models of human adaptation to stress. Thus, our study's Hypothesis 2 was confirmed and Hypothesis 3 was partially confirmed, because only coping perception predicted burnout and control perception did not predict burnout. Nevertheless, these results align with current literature, evidencing the crucial role of cognitive appraisal in understanding stress [6,7,9,36-38] and burnout $[38,39]$. Finally, our results suggest that negative emotions, such as anger, predict higher levels of burnout symptoms, whereas higher levels of positive emotions, such as happiness, predict lower burnout symptoms levels, as suggested in contexts of occupational stress (e.g., [40]). Our results partially confirmed Hypothesis 4 because, from the three negative emotions evaluated in our study (anxiety, dejection, and anger), only one (anger) predicted burnout; also important, Hypothesis 5 was also partially confirmed because, from the two positive emotions evaluated in our study (excitement and happiness), only one (happiness) predicted burnout.
This study has some limitations. We used a critical incident methodology instead of a more appropriate longitudinal design. Although aware of these limitations, it was decided that the critical incident's advantages outweighed its limitations; in fact, we collected data very close in time to the competition event, giving a more realistic vision of how referees adapt to stressful situations. On the other hand, we managed to study a substantial sample, with almost 400 participants, thereby strengthening the results and conclusions drawn.

\section{Conclusions}

This study highlights two pressing needs: theoretically, the need to integrate the variables of stress, cognitive appraisal, emotions, and consequences of stress exposure (in our case, burnout) in order to better understand how referees adapt to competitions; this integration may be the key to advance theory and knowledge about the effects of and reactions to stress. From a practical point of view, our results reinforce the need to reduce the negative effects of stress and burnout by developing intervention programs aimed at increasing referees' patterns of cognitive appraisal based on challenge, coping, and control perceptions, while at the same time reducing threat perceptions. By targeting the cognitive appraisal processes in such programs, referees will develop the tools they need to successfully deal with stressful events, thereby improving their performance, reducing the likelihood of burnout, and promoting an overall positive adaptation to stress.

\section{Competing Interests}

The authors declare that they have no competing interests. 
Citation: Gomes R, Fontes L, Rodrigues M, Dias B (2021) Burnout in Referees: Relations with Stress, Cognitive Appraisal, and Emotions. Int J Psychol Behav Anal 7: 177. doi: https://doi.org/10.15344/2455-3867/2021/177

Page 6 of 7

\section{Author's Contributions}

The authors hereby certify that all authors made substantive intellectual contributions to this study, namely:

1. Substantial contributions to the conception and design, acquisition of data, analysis and interpretation of data.

2. Involved in drafting the manuscript and revising it critically on important intellectual contents

3. Final approval of the version to be published.

\section{Funding}

This study was conducted at the Psychology Research Centre (PSI/01662), School of Psychology, University of Minho, and supported by the Portuguese Foundation for Science and Technology (https://www.fct.pt/index.phtml.pt) and the Portuguese Ministry of Science, Technology and Higher Education (https://www.sec-geral. mec.pt/pt-pt/pagina/ciencia-tecnologia-e-ensino-superior-0; UID/ PSI/01662/2019), through national funds (PIDDAC). The funding entities had no involvement in any part of this work.

\section{References}

1. Ritchie J, Basevitch I, Rodenberg R, Tenenbaum G (2017) Situation criticality and basketball officials' stress levels. J Sports Sci 35: 2080-2087.

2. Silva AI, Rodriguez-Añez CR, Frómeta ER (2002) O árbitro de futebol - Uma abordagem histórico-crítica. Journal of Physical Education 13: 39-45.

3. Alonso-Arbiol I, Arratibel N, Gómez E (2008) La motivación del colectivo arbitral en fútbol: Un estudio cualitativo. Revista de Psicología del Deporte 17: 187-203.

4. Balch MJ, Scott D (2007) Contrary to popular belief, refs are people too Personality and perceptions of officials. Journal of Sport Behavior 30: 3-20.

5. Lazarus RS (1991) Emotion and adaptation. Oxford University Press on Demand.

6. Lazarus RS (1999) Stress and emotion: A new synthesis. Springer Publishing Co.

7. Gomes AR (2014) Positive human functioning in stress situations: An interactive proposal. Resende R \& Albuquerque A (Eds.). Positive human functioning from a multidimensional perspective: Promoting stress adaptation. Nova Science 1: 165-194.

8. Lazarus RS (2000) How emotions influence performance in competitive sports. The Sport Psychologist 14: 229-252.

9. Lazarus RS, Folkman S (1984) Stress, appraisal, and coping. Springer publishing company.

10. Nicholls AR, Levy AR, Jones L, Rengamani M, Polman RC, et al. (2011) An exploration of the two-factor schematization of relation meaning and emotions among professional rugby union players. International Journal of Sport and Exercise Psychology 9: 78-91.

11. Bartholomew KJ, Arnold R, Hampson RJ, Fletcher D (2017) Organizational stressors and basic psychological needs: The mediating role of athletes' appraisal mechanisms. Scand J Med Sci Sports 27: 2127-2139.

12. McGreary M, Morris R, Whitehead A (2020) Thinking aloud: Stress and coping in junior cricket batsmen during challenge and threat states. Perceptual and Motor Skills 127: 1095-1117.

13. Hill DM, Matthews N, Senior R (2016) The psychological characteristics of performance under pressure in professional rugby union referees. The Sport Psychologist 30: 376-387.

14. Neil R, Bayston P, Hanton S, Wilson K (2013) The influence of stress and emotions on association football referees' decision-making. Sport \& Exercise Psychology Review 9: 22-41.

15. Armon G, Shirom A, Melamed S (2012) The Big Five personality factors as predictors of changes across time in burnout and its facets. J Pers 80: 403427.
16. Maslach C, Leiter MP, Jackson SE (2012) Making a significant difference with burnout interventions: Researcher and practitioner collaboration. Journal of Organizational Behavior 33: 296-300.

17. Shirom A, Melamed S (2006) A comparison of the construct validity of two burnout measures in two groups of professionals. International Journal of Stress Management 13: 176

18. Simmons N (2018) Critical Incident Method. In M. Allen (Ed.), The SAGE encyclopedia of communication research methods. Thousand Oaks: SAGE Publications, Inc.

19. Gomes AR (2017) Questionário de Stress em Árbitros (QSA).Unpublished technical report. School of Psychology, University of Minho.

20. Bentler PM (2007) On tests and indices for evaluating structural models. Personality and Individual Differences 42: 825-829.

21. Gomes $A R$, Teixeira $F$ (2013) Influência dos processos de avaliação cognitiva na atividade laboral de bombeiros portugueses. Psico-USF 18: 309-319.

22. Jones MV, Lane AM, Bray SR, Uphill M, Catlin J, et al. (2005) Development and validation of the Sport Emotion Questionnaire. Journal of Sport and Exercise Psychology 27: 407-431.

23. Shirom A (1989) Burnout in work organizations. In CL Cooper \& I Robertson (Eds.), International review of industrial and organizational psychology. Wiley.

24. Gomes AR, Teixeira P (2016) Stress, cognitive appraisal, and psychological health: Testing instruments for health professionals. Stress Health 32: 167172.

25. Blascovich J (2008) Challenge and threat. In A.J. Elliot (Ed.), Handbook of approach and avoidance motivation. Psychology Press.

26. Johansen BT, Haugen T (2013) Anxiety level and decision-making among Norwegian top-class soccer referees. International Journal of Sport and Exercise Psychology, 11: 215-226.

27. Turner MJ, Jones MV (2014) Stress, emotions and athletes' positive adaptation to sport: Contributions from a transactional perspective. In $R$ Resende \& A Albuquerque (Eds), Positive human functioning from a multidimensional perspective. Nova Science Publishers.

28. Wolfson S, Neave N (2007) Coping under pressure: Cognitive strategies for maintaining confidence among soccer referees. Journal of Sport Behavior 30: 232-247

29. Stewart MJ, Ellery PJ, Ellery J, Maher L (2004) Perceived psychological stress among high school basketball officials. Percept Mot Skills 99: 463-469.

30. Gustafsson H, Sagar SS, Stenling A (2017) Fear of failure, psychological stress, and burnout among adolescent athletes competing in high level sport. Scand J Med Sci Sports 27: 2091-2102.

31. Al-Haliq M, Altahayneh ZL, Oudat ME (2014) Levels of burnout among sports referees in Jordan. Journal of Physical Education and Sport 14: 47.

32. Oliveira ABD, Penna EM, Pires DA (2018) Síndrome de burnout em árbitros de futebol. Revista de Psicología del Deporte 27: 31-36.

33. Pedrosa I, García-Cueto E (2016) Síndrome de Burnout en árbitros de élite: la liga de fútbol profesional española (LFP) a estudio. Revista Iberoamericana de Diagnóstico y Evaluación e Avaliação Psicológica, Lisboa 42: 59-68.

34. Rainey DW (1995) Stress, burnout, and intention to terminate among umpires. Journal of Sport Behavior 18: 312-323.

35. Rainey DW, Hardy L (1999) Sources of stress, burnout and intention to terminate among rugby union referees. J Sports Sci 17: 797-806.

36. Gomes AR (2013) Adaptação humana no desporto: Uma perspetiva transacional. In D Bartholomeu, JM Montiel, FK Miguel, LF Carvalho, JMH Bueno (Eds.), Atualização em avaliação e tratamento das emoções. Vetor Editora.

37. Nicholls AR, Perry JL, Calmeiro L (2014) Precompetitive achievement goals, stress appraisals, emotions, and coping among athletes. J Sport Exerc Psychol 36: 433-445.

38. Vilela C, Gomes AR (2015) Ansiedade, avaliação cognitiva e esgotamento na formação desportiva: Estudo com jovens atletas. Motricidade 11: 104-120.

39. Cremades JG, Wated G, Wiggins MS (2011) Multiplicative measurements of a trait anxiety scale as predictors of burnout. Measurement in Physical Education and Exercise Science 15: 220-233. 
Citation: Gomes R, Fontes L, Rodrigues M, Dias B (2021) Burnout in Referees: Relations with Stress, Cognitive Appraisal, and Emotions. Int J Psychol Behav Anal 7: 177. doi: https://doi.org/10.15344/2455-3867/2021/177

Page 7 of 7

40. Szczygiel DD, Mikolajczak M (2018) Emotional intelligence buffers the effects of negative emotions on job burnout in nursing. Front Psychol 9: 2649. 